\title{
Some properties of the Mittag-Leffler functions and their relation with the Wright functions
}

\author{
Muhammet Kurulay ${ }^{1,2^{*}}$ and Mustafa Bayram²
}

\section{"Correspondence:}

mkurulay@yildiz.edu.tr

'Department of Mathematics,

University of Connecticut, 196

Auditorium Road, U-3009Storrs,

Mansfield, CT 06269-3009, USA

2Department of Mathematics,

Faculty of Art and Sciences, Yildiz

Technical University,

34210-Davutpasa, Istanbul, Turkey

\begin{abstract}
This paper is a short description of our recent results on an important class of the so-called Mittag-Leffler functions, which became important as solutions of fractional order differential and integral equations, control systems and refined mathematical models of various physical, chemical, economical, management and bioengineering phenomena. We have studied the Mittag-Leffler functions as their typical representatives, including many interesting special cases that have already proven their usefulness in fractional calculus and its applications. We obtained a number of useful relationships between the Mittag-Leffler functions and the Wright functions. The Wright function plays an important role in the solution of a linear partial differential equation. The Wright function, which we denote by $W(z ; \alpha, \beta)$, is so named in honor of Wright who introduced and investigated this function in a series of notes starting from 1933 in the framework of the asymptotic theory of partitions. MSC: $33 \mathrm{E} 12$
\end{abstract}

Keywords: Mittag-Leffler functions; the Wright functions

\section{Introduction}

\subsection{The Mittag-Leffler function}

The Mittag-Leffler function is an important function that finds widespread use in the world of fractional calculus. Just as the exponential naturally arises out of the solution to integer order differential equations, the Mittag-Leffler function plays an analogous role in the solution of non-integer order differential equations. In fact, the exponential function itself is a very special form, one of an infinite set of these seemingly ubiquitous functions. The standard definition of Mittag-Leffler [1] is given as follows:

$$
E_{\alpha}(z)=\sum_{k=0}^{\infty} \frac{z^{k}}{\Gamma(\alpha k+1)}, \quad \alpha \in C, R(\alpha)>0, z \in C .
$$

A two-parameter function of the M-L (Mittag-Leffler) type is defined by the series expansion [2]

$$
E_{\alpha, \beta}(z)=\sum_{k=0}^{\infty} \frac{z^{k}}{\Gamma(\alpha k+\beta)} \quad(\alpha, \beta \in C, R(\alpha)>0, R(\beta)>0, z \in C)
$$

\section{Springer}

(C) 2012 Kurulay and Bayram; licensee Springer. This is an Open Access article distributed under the terms of the Creative Commons Attribution License (http://creativecommons.org/licenses/by/2.0), which permits unrestricted use, distribution, and reproduction in any medium, provided the original work is properly cited. 
The M-L function provides a simple generalization of the exponential function because of the substitution of $n !=\Gamma(n+1) n$ ! with $(n \alpha) !=\Gamma(n \alpha+1)$. Particular cases of (2) recover elementary functions are recovered

$$
E_{1,2}=\frac{e^{z}-1}{z}, \quad E_{2,2}\left(z^{2}\right)=\frac{\sinh (z)}{z},
$$

and

$$
E_{1 / 2,1}=e^{z^{2}} \operatorname{erfc}(-z)
$$

where erf (erfc) denotes the (complementary) error function defined as [3]

$$
\operatorname{erfc}(z)=\frac{2}{\sqrt{\pi}} \int_{z}^{\infty} e^{-t^{2}} d t
$$

By means of the series representation, a generalization of (1) and (2) is introduced by Prabhakar [4] as

$$
E_{\alpha, \beta}^{\gamma}(z)=\sum_{k=0}^{\infty} \frac{(\gamma)_{n}}{\Gamma(\alpha k+\beta)} \cdot \frac{z^{k}}{k !} \quad(\alpha, \beta, \gamma \in C, R(\alpha)>0, R(\beta), R(\gamma)>0, z \in C),
$$

where $(\gamma)_{n}$ is the Pochhammer symbol [5] given by

$$
(\lambda)_{n}=\frac{\Gamma(\lambda+n)}{\Gamma(\lambda)}= \begin{cases}1 & (n=0 ; \lambda \neq 0), \\ (\gamma)_{n}=\gamma(\gamma+1)(\gamma+2) \cdots(\gamma+n-1) & (n \in N ; \lambda \in C) .\end{cases}
$$

Note that

$$
E_{1,1}^{1}(z)=e^{z}, \quad E_{\alpha, 1}^{1}(z)=E_{\alpha}(z), \quad E_{\alpha, \beta}^{1}(z)=E_{\alpha, \beta}(z) .
$$

Some new properties of the Mittag-Leffler function, including a definite integral and recurrence relation, were investigated in $[6,7]$.

\subsection{The Wright function}

The Wright function plays an important role in the solution of a linear partial differential equation. The Wright function, which we denote by $W(z ; \alpha, \beta)$, is so named in honor of Wright, who introduced and investigated this function in a series of notes starting from 1933 in the framework of the asymptotic theory of partitions. This function was introduced that related Mittag-Leffler [8-11]. We obtained a number of useful relationships between the Mittag-Leffler functions and the Wright functions.

\subsubsection{Definition}

The Wright function is defined by the series representation, convergent in the whole zcomplex plane [12]

$$
W(z ; \alpha, \beta)=\sum_{k=0}^{\infty} \frac{z^{k}}{k ! \Gamma(\alpha k+\beta)} .
$$




\subsubsection{The integral representation of the Wright function}

$$
W(z ; \alpha, \beta)=\frac{1}{2 \pi i} \int_{H a} \exp \left\{u+z u^{-\alpha}\right\} u^{-\beta} d u,
$$

where Ha denotes the Hankel path. To prove the Hankel path, let us write the integrated function in the form of a power series in $\mathrm{z}$ and perform term-by-term integration using the integral representation formula for the reciprocal gamma function

$$
\frac{1}{\Gamma(z)}=\int_{H a} e^{\zeta} \zeta^{z} d \zeta
$$

In fact,

$$
\begin{aligned}
W(z ; \alpha, \beta) & =\frac{1}{2 \pi i} \int_{H a} \exp \left\{u+z u^{-\alpha}\right\} u^{-\beta} d u=\frac{1}{2 \pi i} \int_{H a} e^{u}\left[\sum_{n=0}^{\infty} \frac{z^{n}}{n !} u^{-\alpha n}\right] u^{-\beta} d u \\
& =\sum_{n=0}^{\infty} \frac{z^{n}}{n !}\left[\frac{1}{2 \pi i} \int_{H a} e^{u} u^{-\alpha n-\beta} d u\right]=\sum_{n=0}^{\infty} \frac{z^{n}}{n ! \Gamma(\alpha n+\beta)} .
\end{aligned}
$$

\subsubsection{The Laplace transform of the Wright function}

We recall that the Mittag-Leffler function plays fundamental roles in applications of fractional calculus like fractional relaxation and fractional oscillation [13-16]. Kiryakova introduced and studied the multi-index Mittag-Leffler functions as their typical representatives, including many interesting special cases that have already proven their usefulness in FC and its applications [17]. Srivatava and Tomovski introduced and investigated the fractional calculus with an integral operator which contains the following family of generalized Mittag-Leffler functions [18]. Haubold, Mathaian and Saxena studied the Mittag-Leffler functions and their applications [19]. There is an interesting link between the Wright function and the Mittag-Leffler function. We now point out that the Wright function is related to the Mittag-Leffler function through the following Laplace transform pair:

$$
\begin{aligned}
L\{W(t ; \alpha, \beta) ; s\} & =L\left\{\sum_{k=0}^{\infty} \frac{t^{k}}{k ! \Gamma(\alpha k+\beta)} ; s\right\} \\
& =\sum_{k=0}^{\infty} \frac{1}{k ! \Gamma(\alpha k+\beta)} \cdot \frac{1}{s^{k+1}} \\
& =s^{-1} E_{\alpha, \beta}\left(s^{-1}\right) .
\end{aligned}
$$

\section{Some properties of the Mittag-Leffler functions}

Theorem 1 (Derivative of the Mittag-Leffler function) If $\alpha, \beta, \gamma \in C, R(\alpha)>0, R(\beta)>0$, $R(\gamma)>0, z \in C$ and $r, n \in N$, then

$$
\frac{d^{n}}{d x^{n}}\left[z^{\beta-1} E_{\alpha, \beta+r \alpha}^{\gamma}(z)\right]=z^{\beta-n-1} E_{\alpha, \beta+\gamma \alpha-n}^{\gamma}(z) .
$$

Proof Using definition (3), we have that

$$
\frac{d^{n}}{d x^{n}}\left[z^{\beta-1} E_{\alpha, \beta+r \alpha}^{\gamma}(z)\right]=\sum_{k=0}^{\infty} \frac{(\gamma)_{k}}{\Gamma(\alpha(k+r)+\beta-n)} \frac{z^{k+\beta-1-n}}{k !}=z^{\beta-n-1} E_{\alpha, \beta+r \alpha-n}^{\gamma}(z) .
$$


The Wright function is expressed with help of the Mittag-Leffler function:

$$
\frac{d^{n}}{d x^{n}}\left[z^{\beta-1} E_{\alpha, \beta+r \alpha}^{\gamma}(z)\right]=\sum_{k=0}^{\infty} z^{\beta-1-n}(\gamma)_{k} W(z ; \alpha, \beta+r \alpha-n) .
$$

Theorem 2 (Integration of the Mittag-Leffler function) If $\alpha, \beta, \gamma \in C, R(\alpha)>0, R(\beta)>0$, $R(\gamma)>0, z \in C$ and $r \in N$, then

$$
\int_{0}^{z} z^{\beta+r \alpha-1} E_{\alpha, \beta+r \alpha}^{\gamma}(z) d z=z^{\beta+r \alpha} E_{\alpha, \beta+r \alpha+1}^{\gamma}(z) .
$$

Proof According to the Mittag-Leffler function, we have

$$
z^{\beta+r \alpha-1} E_{\alpha, \beta+r \alpha}^{\gamma}(z)=\sum_{k=0}^{\infty} \frac{(\gamma)_{k}}{\Gamma(\alpha(k+r)+\beta)} \cdot \frac{z^{k+r \alpha+\beta-1}}{k !} .
$$

Integrating both sides gives

$$
\begin{aligned}
\int_{0}^{z} z^{\beta+r \alpha-1} E_{\alpha, \beta+r \alpha}^{\gamma}(z) d z & =\int_{0}^{z} \sum_{k=0}^{\infty} \frac{(\gamma)_{k}}{\Gamma(\alpha(k+r)+\beta)} \cdot \frac{z^{k+r \alpha+\beta-1}}{k !} d z \\
& =\sum_{k=0}^{\infty} \frac{(\gamma)_{k}}{\Gamma(\alpha(k+r)+\beta+1)} \cdot \frac{z^{k+r \alpha+\beta}}{k !} \\
& =z^{\beta+r \alpha} E_{\alpha, \beta+r \alpha+1}^{\gamma}(z) .
\end{aligned}
$$

Relation with the Wright functions is as follows:

$$
\begin{aligned}
\int_{0}^{z} z^{\beta+r \alpha-1} E_{\alpha, \beta+r \alpha}^{\gamma}(z) d z & =\int_{0}^{z} \sum_{k=0}^{\infty} \frac{(\gamma)_{k}}{\Gamma(\alpha(k+r)+\beta)} \cdot \frac{z^{k+r \alpha+\beta-1}}{k !} d z \\
& =\sum_{k=0}^{\infty} z^{\beta+r \alpha}(\gamma)_{k} W(z ; \alpha, \beta+r \alpha+1) .
\end{aligned}
$$

Theorem 3 Let $\alpha, \beta, \gamma \in C, R(\alpha)>0, R(\beta)>0, R(\gamma)>0, z \in C, r \in N$, then

$$
E_{\alpha, \beta}^{\gamma}(z)=\beta E_{\alpha, \beta+1}^{\gamma}(z)+\alpha z \frac{d}{d z} E_{\alpha, \beta+1}^{\gamma}(z)
$$

Proof By definition (3), we have that

$$
\begin{aligned}
\beta E_{\alpha, \beta+1}^{\gamma}(z)+\alpha z \frac{d}{d z} E_{\alpha, \beta+1}^{\gamma}(z) & =\beta E_{\alpha, \beta+1}^{\gamma}(z)+\alpha z \frac{d}{d z} \sum_{n=0}^{\infty} \frac{(\gamma)_{n}}{\Gamma(\alpha n+\beta+1)} \cdot \frac{z^{n}}{n !} \\
& =\sum_{n=0}^{\infty} \frac{\beta(\gamma)_{n}}{\Gamma(\alpha n+\beta+1)} \cdot \frac{z^{n}}{n !}+\sum_{n=0}^{\infty} \frac{\alpha n(\gamma)_{n}}{\Gamma(\alpha n+\beta+1)} \cdot \frac{z^{n}}{n !} \\
& =E_{\alpha, \beta}^{\gamma}(z) .
\end{aligned}
$$


Relation with the Wright functions is as follows:

$$
\begin{aligned}
\beta E_{\alpha, \beta+1}^{\gamma}(z)+\alpha z \frac{d}{d z} E_{\alpha, \beta+1}^{\gamma}(z) & =\beta E_{\alpha, \beta+1}^{\gamma}(z)+\alpha z \frac{d}{d z} \sum_{n=0}^{\infty} \frac{(\gamma)_{n}}{\Gamma(\alpha n+\beta+1)} \cdot \frac{z^{n}}{n !} \\
& =\sum_{n=0}^{\infty} \frac{\beta(\gamma)_{n}}{\Gamma(\alpha n+\beta+1)} \cdot \frac{z^{n}}{n !}+\sum_{n=0}^{\infty} \frac{\alpha n(\gamma)_{n}}{\Gamma(\alpha n+\beta+1)} \cdot \frac{z^{n}}{n !} \\
& =\sum_{n=0}^{\infty}(\gamma)_{n} W(z ; \alpha, \beta) .
\end{aligned}
$$

Theorem 4 For $\alpha, \beta, \gamma \in C, R(\alpha)>0, R(\beta)>0, R(\gamma)>0, z \in C, r \in N$. 'Note that'

$$
\begin{aligned}
& E_{\alpha, \beta+r \alpha+1}^{\gamma}(z)-E_{\alpha, \beta+r \alpha+2}^{\gamma}(z) \\
& =(\beta+r \alpha)(\beta+r \alpha+2) E_{\alpha, \beta+r \alpha+3}^{\gamma}(z)+\alpha^{2} z^{2} \frac{d^{2}}{d z^{2}} E_{\alpha, \beta+r \alpha+3}^{\gamma}(z) \\
& \quad+\alpha\{\alpha+2+2(\beta+r \alpha)\} z \frac{d}{d z} E_{\alpha, \beta+r \alpha+3}^{\gamma}(z) .
\end{aligned}
$$

Proof We have

$$
\begin{aligned}
& E_{\alpha, \beta+r \alpha+1}^{\gamma}(z)=\sum_{k=0}^{\infty} \frac{(\gamma)_{k}}{(\alpha(k+r)+\beta) \Gamma(\alpha(k+r)+\beta)} \cdot \frac{z^{k}}{k !}, \\
& E_{\alpha, \beta+r \alpha+2}^{\gamma}(z)=\sum_{k=0}^{\infty} \frac{(\gamma)_{k}}{(\alpha(k+r)+\beta)(\alpha(k+r)+\beta+1) \Gamma(\alpha(k+r)+\beta)} \cdot \frac{z^{k}}{k !} .
\end{aligned}
$$

Equation (6) can be written as follows:

$$
\begin{aligned}
E_{\alpha, \beta+r \alpha+2}^{\gamma}(z) & =\sum_{k=0}^{\infty}\left[\frac{1}{\alpha(k+r)+\beta}-\frac{1}{\alpha(k+r)+\beta+1}\right] \frac{z^{k}(\gamma)_{k}}{\Gamma(\alpha(k+r)+\beta) k !} \\
& =E_{\alpha, \beta+r \alpha+1}^{\gamma}(z)-\sum_{k=0}^{\infty} \frac{z^{k}(\gamma)_{k}}{(\alpha(k+r)+\beta+1) \Gamma(\alpha(k+r)+\beta) k !} .
\end{aligned}
$$

We find from equation (7) that

$$
\begin{aligned}
E_{\alpha, \beta+r \alpha+1}^{\gamma}(z)-E_{\alpha, \beta+r \alpha+2}^{\gamma}(z)= & \sum_{k=0}^{\infty} \frac{z^{k}(\gamma)_{k}}{(\alpha(k+r)+\beta+1) \Gamma(\alpha(k+r)+\beta) k !} \\
= & \frac{z^{k}(\gamma)_{k}}{k !}\left(\frac{1}{(\alpha(k+r)+\beta+2)(\alpha(k+r)+\beta+1) \Gamma(\alpha(k+r)+\beta)}\right. \\
& \left.+\frac{1}{(\alpha(k+r)+\beta+2) \Gamma(\alpha(k+r)+\beta)}\right)
\end{aligned}
$$

or

$$
\begin{aligned}
& \sum_{k=0}^{\infty} \frac{z^{k}(\gamma)_{k}}{(\alpha(k+r)+\beta+1) \Gamma(\alpha(k+r)+\beta) k !} \\
& \quad=\sum_{k=0}^{\infty} \frac{(\alpha(k+r)+\beta) z^{k}(\gamma)_{k}}{\Gamma(\alpha(k+r)+\beta+3) k !}+\sum_{k=0}^{\infty} \frac{(\alpha(k+r)+\beta)(\alpha(k+r)+\beta+1) z^{k}(\gamma)_{k}}{\Gamma(\alpha(k+r)+\beta+3) k !}
\end{aligned}
$$




$$
\begin{aligned}
= & \sum_{k=0}^{\infty}\left\{\frac{z^{k}(\gamma)_{k}}{k !}\left(\frac{\alpha k}{\Gamma(\alpha(k+r)+\beta+3)}+\frac{(\beta+r \alpha)}{\Gamma(\alpha(k+r)+\beta+3)}\right)\right\} \\
& +\sum_{k=0}^{\infty}\left\{\frac { z ^ { k } ( \gamma ) _ { k } } { k ! } \left(\frac{(k \alpha)^{2}}{\Gamma(\alpha(k+r)+\beta+3)}\right.\right. \\
& \left.\left.+\frac{k \alpha(2 \beta+2 r \alpha+1)}{\Gamma(\alpha(k+r)+\beta+3)}+\frac{(\beta+r)^{2}+(\beta+r)}{\Gamma(\alpha(k+r)+\beta+3)}\right)\right\} .
\end{aligned}
$$

We now say each summation on the right-hand side of equation (9) is as follows:

$$
\begin{aligned}
\frac{d^{2}}{d z^{2}}\left\{z^{2} E_{\alpha, \beta+\gamma \alpha+3}^{\gamma}(z)\right\}= & \sum_{k=0}^{\infty} \frac{(k+2)(k+1)(\gamma)_{k}}{\Gamma(\alpha(k+r)+\beta+3)} \cdot \frac{z^{k}}{k !} \\
= & \sum_{k=0}^{\infty} \frac{k^{2}(\gamma)_{k}}{\Gamma(\alpha(k+r)+\beta+3)} \cdot \frac{z^{k}}{k !} \\
& +3 \sum_{k=0}^{\infty} \frac{k(\gamma)_{k}}{\Gamma(\alpha(k+r)+\beta+3)} \cdot \frac{z^{k}}{k !}+2 E_{\alpha, \beta+r \alpha+3}^{\gamma}(z)
\end{aligned}
$$

or

$$
\begin{aligned}
\sum_{k=0}^{\infty} & \frac{k^{2}(\gamma)_{k}}{\Gamma(\alpha(k+r)+\beta+3)} \cdot \frac{z^{k}}{k !} \\
= & 4 z \frac{d}{d z} E_{\alpha, \beta+r \alpha+3}^{\gamma}(z) \\
& \quad+z^{2} \frac{d^{2}}{d z^{2}} E_{\alpha, \beta+r \alpha+3}^{\gamma}(z)-3 \sum_{k=0}^{\infty} \frac{k(\gamma)_{k}}{\Gamma(\alpha(k+r)+\beta+3)} \cdot \frac{z^{k}}{k !} .
\end{aligned}
$$

We find from equation (11) that

$$
\sum_{k=0}^{\infty} \frac{k^{2}(\gamma)_{k}}{\Gamma(\alpha(k+r)+\beta+3)} \cdot \frac{z^{k}}{k !}=z \frac{d}{d z} E_{\alpha, \beta+\gamma \alpha+3}^{\gamma}(z)+z^{2} \frac{d^{2}}{d z^{2}} E_{\alpha, \beta+r \alpha+3}^{\gamma}(z)
$$

Using equations (9), (11), and (12), we get

$$
\begin{aligned}
& E_{\alpha, \beta+r \alpha+1}^{\gamma}(z)-E_{\alpha, \beta+r \alpha+2}^{\gamma}(z) \\
& =(\beta+r \alpha)(\beta+r \alpha+2) E_{\alpha, \beta+r \alpha+3}^{\gamma}(z)+\alpha^{2} z^{2} \frac{d^{2}}{d z^{2}} E_{\alpha, \beta+r \alpha+3}^{\gamma}(z) \\
& \quad+\alpha\{\alpha+2+2(\beta+r \alpha)\} z \frac{d}{d z} E_{\alpha, \beta+r \alpha+3}^{\gamma}(z) .
\end{aligned}
$$

Relation with the Wright functions is as follows:

$$
\begin{aligned}
& E_{\alpha, \beta+r \alpha+1}^{\gamma}(z)-E_{\alpha, \beta+r \alpha+2}^{\gamma}(z) \\
& \quad=\sum_{k=0}^{\infty}(\beta+r \alpha)(\beta+r \alpha+2)(\gamma)_{k} W(z ; \alpha, \beta+r \alpha+3)
\end{aligned}
$$




$$
\begin{aligned}
& +\sum_{k=0}^{\infty} \alpha^{2} z^{2}(\gamma)_{k} \frac{d^{2}}{d z^{2}} W(z ; \alpha, \beta+r \alpha+3) \\
& +\sum_{k=0}^{\infty} \alpha\{\alpha+2+2(\beta+r \alpha)\}(\gamma)_{k} z \frac{d}{d z} W(z ; \alpha, \beta+r \alpha+3) .
\end{aligned}
$$

Theorem 5 If $\alpha, \beta, \gamma \in C, R(\alpha)>0, R(\beta)>0, R(\gamma)>0, z \in C, r \in N$, then

$$
z^{r} E_{\alpha, \beta+r \alpha}^{\gamma}(z)=E_{\alpha, \beta}^{\gamma}(z)-\sum_{k=0}^{r-1} \frac{(\gamma)_{k}}{\Gamma(k \alpha+\beta)} \frac{z^{k}}{k !}
$$

Proof We have from (13)

$$
\sum_{k=r}^{\infty} \frac{(\gamma)_{k}}{\Gamma(k \alpha+\beta)} \frac{z^{k}}{k !}=E_{\alpha, \beta}^{\gamma}(z)-\sum_{k=0}^{r-1} \frac{(\gamma)_{k}}{\Gamma(k \alpha+\beta)} \frac{z^{k}}{k !}
$$

For $r=2,3,4, \ldots$, we obtain

$$
\begin{aligned}
& z^{2} E_{\alpha, \beta+2 \alpha}^{\gamma}(z)=E_{\alpha, \beta}^{\gamma}(z)-\frac{1}{\Gamma(\beta)}-\frac{(\gamma)_{1} z}{\Gamma(\alpha+\beta)}, \\
& z^{3} E_{\alpha, \beta+3 \alpha}^{\gamma}(z)=E_{\alpha, \beta}^{\gamma}(z)-\frac{1}{\Gamma(\beta)}-\frac{(\gamma)_{1} z}{\Gamma(\alpha+\beta)}-\frac{(\gamma)_{2} z^{2}}{\Gamma(2 \alpha+\beta)}, \\
& z^{4} E_{\alpha, \beta+4 \alpha}^{\gamma}(z)=E_{\alpha, \beta}^{\gamma}(z)-\frac{1}{\Gamma(\beta)}-\frac{(\gamma)_{1} z}{\Gamma(\alpha+\beta)}-\frac{(\gamma)_{2} z^{2}}{\Gamma(2 \alpha+\beta)}-\frac{(\gamma)_{3} z^{3}}{\Gamma(3 \alpha+\beta)}, \\
& \vdots \\
& \sum_{k=r}^{\infty} \frac{(\gamma)_{k}}{\Gamma(k \alpha+\beta)} \frac{z^{k}}{k !}=E_{\alpha, \beta}^{\gamma}(z)-\sum_{k=o}^{r-1} \frac{(\gamma)_{k}}{\Gamma(k \alpha+\beta)} \frac{z^{k}}{k !} .
\end{aligned}
$$

Relation with the Wright functions is as follows:

$$
\sum_{k=r}^{\infty} \frac{(\gamma)_{k}}{\Gamma(k \alpha+\beta)} \frac{z^{k}}{k !}=\sum_{k=0}^{\infty}(\gamma)_{k} W(z ; \alpha, \beta)-\sum_{k=0}^{r-1}(\gamma)_{k} W(z ; \alpha, \beta)
$$

\section{Competing interests}

The authors declare that they have no competing interests.

\section{Authors' contributions}

MK defined the research theme. MK designed theorems and proofs, analyzed the data, interpreted the results and wrote the paper. MB participated drafted the manuscript. All authors read and approved the final manuscript.

\section{Acknowledgements}

This work was supported by the scientific and technological research council of Turkey (TUBITAK).

Received: 6 August 2012 Accepted: 4 October 2012 Published: 17 October 2012

\section{References}

1. Mittag-Leffler, G: Sur la nouvelle fontion $E_{\alpha}$. Comptes Rendus Hebdomadaires Des Seances Del Academie Des Sciences, Paris 2 137, 554-558 (1903)

2. Wiman, A: Über den fundamental Satz in der Theorie der Funktionen. Acta Math. 29, 191-201 (1905)

3. Podlubny, I: Fractional Differential Equations. Academic Press, San Diego (1999) 
4. Prabhakar, TR: A singular integral equation with a generalized Mittag-Leffler function in the kernel. Yokohama Math. J. 19, 7-15 (1971)

5. Rainville, ED: Special Functions. Macmillan, New York (1960)

6. Gupta, IS, Debnath, L: Some properties of the Mittag-Leffer functions. Integral Transforms Spec. Funct. 18(5), 329-336 (2007)

7. Peng, J, Li, K: A note on property of the Mittag-Leffler function. J. Math. Anal. Appl. 370(2), 635-638 (2010)

8. Wright, EM: On the coefficients of power series having exponential singularities. J. Lond. Math. Soc. 8, 71-79 (1933)

9. Wright, EM: The asymptotic expansion of the generalized Bessel function. Proc. Lond. Math. Soc. 38, 257-270 (1935)

10. Wright, EM: The asymptotic expansion of the generalized hypergeometric function. J. Lond. Math. Soc. 10, 287-293 (1935)

11. Wright, EM: The generalized Bessel function of order greater than one. Q. J. Math., Oxford Ser. 11, 36-48 (1940)

12. Erdelyi, A, Magnus, W, Oberhettinger, F, Tricomi, FG: Higher Transcendental Functions, vol. 3, chapter 18. McGraw-Hill, New York (1954)

13. Mainardi, F, Mura, A, Pagnini, G: The M-Wright function in time-fractional diffusion processes: a tutorial survey. Int. J. Differ. Equ., (2010). doi:10.1155/2010/104505

14. Mainardi, F, Gorenflo, R: Time-fractional derivatives in relaxation processes: a tutorial survey. Fract. Calc. Appl. Anal. 10 269-308 (2007)

15. Achar, BNN, Hanneken, JW, Clarke, T: Damping characteristics of a fractional oscillator. Physica A 339, $311-319$ (2004)

16. Mainardi, F: Fractional Calculus and Waves in Linear Viscoelasticity. Imperial College Press, London (2010)

17. Kiryakova, $\vee$ : The multi-index Mittag-Leffler functions as an important class of special functions of fractional calculus. Comput. Math. Appl. 59, 1885-1895 (2010)

18. Srivastava, HM, Tomovski, Ž: Fractional calculus with an integral operator containing a generalized Mittag-Leffler function in the kernel. Appl. Math. Comput. 211, 198-210 (2009)

19. Haubold, HJ, Mathaiand, AM, Saxena, RK: Mittag-Leffler functions and their applications. J. Appl. Math. 2011, 298628 (2011). doi:10.1155/2011/298628

doi:10.1186/1687-1847-2012-181

Cite this article as: Kurulay and Bayram: Some properties of the Mittag-Leffler functions and their relation with the Wright functions. Advances in Difference Equations 2012 2012:181.

\section{Submit your manuscript to a SpringerOpen ${ }^{\circ}$ journal and benefit from:}

- Convenient online submission

Rigorous peer review

- Immediate publication on acceptance

- Open access: articles freely available online

- High visibility within the field

- Retaining the copyright to your article 